\title{
Stability of Pseudospectral and Finite-Difference Methods for Variable Coefficient Problems
}

\author{
By David Gottlieb*, Steven A. Orszag**, and Eli Turkel***
}

\begin{abstract}
It is shown that pseudospectral approximation to a special class of variable coefficient one-dimensional wave equations is stable and convergent even though the wave speed changes sign within the domain. Computer experiments indicate similar results are valid for more general problems. Similarly, computer results indicate that the leapfrog finite-difference scheme is stable even though the wave speed changes sign within the domain. However, both schemes can be asymptotically unstable in time when a fixed spatial mesh is used.
\end{abstract}

1. Introduction. The semidiscrete Fourier (pseudospectral) approximation to the differential equation

$$
u_{t}=c(x) u_{x}, \quad u(x+2 \pi)=u(x), \quad 0 \leqslant x \leqslant 2 \pi, t>0,
$$

is given by the following algorithm [3], [8], [11].

(1) Construct the trigonometric interpolant $u_{N}$ of $u(x, t)$ at the points $x_{j}=j \pi / N$, $j=0,1, \ldots, 2 N-1$. Hence,

$$
u_{N}\left(x_{j}\right)=\sum_{k=-N}^{N} a_{k}(t) e^{i k x_{j}}
$$

where

$$
a_{k}(t)=\frac{1}{2 N c_{k}} \sum_{j=0}^{2 N-1} u\left(x_{j}, t\right) e^{-i j x_{k}}, \quad-N<k<N
$$

where $c_{k}=1, k \neq \pm N, c_{N}=c_{-N}=2$.

Furthermore, since $u$ is real we have $a_{k}=a_{-k}^{*}$ for $-(N-1)<k<N-1$ and also $a_{N}=a_{-N}$ is real. Note that $u_{N}\left(x_{j}, t\right)=u\left(x_{j}, t\right)$.

(2) Differentiating (1.2), we have that

$$
\frac{\partial u_{N}}{\partial x}\left(x_{j}\right)=\sum_{k=-N}^{N} i k a_{k}(t) e^{i k x_{j}}
$$

Received January 16, 1980; revised August 18, 1980 and February 19, 1981.

1980 Mathematics Subject Classification. Primary 65M10, 65M99.

* Work supported by NASA Contract No. NAS 1-14101 at ICASE, NASA Langley Research Center, Hampton, Virginia and AFOSR Grant No. 77-3405.

** Work supported by AFOSR Grant No. 77-3405 and NSF Grant No. ATM-7817092.

*** Work supported by NASA Contract No. NAS 1-14101 at ICASE, NASA Langley Research Center, Hampton, Virginia. 
Substituting (1.4) into (1.1), we arrive at the semidiscrete approximation

$$
\frac{\partial u_{N}}{\partial t}\left(x_{j}, t\right)=c\left(x_{j}\right) \frac{\partial u_{N}}{\partial x}\left(x_{j}, t\right)
$$

In practice $\partial u_{N} / \partial x$ is calculated using two fast Fourier transforms to evaluate (1.3) and (1.4).

(3) Equation (1.5) is advanced in time by some discretization (see, e.g., [3], [5], [12]).

It is also possible to formulate the algorithm without reference to the Fourier transform. Define

$$
D_{N}(u)=\frac{1}{2 N} \sin N u \cot \frac{u}{2}=\frac{1}{2 N} \sum_{k=-N}^{N} e^{i k u}-\frac{1}{2 N} \cos N u .
$$

$D_{N}(u)$ is a trigonometric polynomial of the form (1.2). Moreover, since $D_{N}\left(x_{k}-x_{j}\right)$ $=\delta_{j k}$, it follows that

$$
u_{N}(x)=\sum_{j=0}^{2 N-1} u\left(x_{j}\right) D_{N}\left(x-x_{j}\right)
$$

When $u_{N}(x)$ is a trigonometric polynomial with degree less than or equal to $N$, the representation (1.7) is unique. Differentiating (1.7), we have

$$
\frac{\partial u_{N}}{\partial x}=\sum_{j=0}^{2 N-1} u\left(x_{j}\right) \frac{\partial}{\partial x} D_{N}\left(x-x_{j}\right)
$$

In particular,

$$
\frac{\partial u_{N}}{\partial x}\left(x_{j}\right)=\frac{1}{2} \sum_{\substack{k=0 \\ k \neq j}}^{2 N-1} u_{N}\left(x_{k}\right)(-1)^{k+j} \cot \left(\frac{x_{j}-x_{k}}{2}\right) .
$$

This formula for $\partial u_{N} / \partial x$ replaces (1.3)-(1.4) in (1.5). A comparison of the computational efficiency of (1.3)-(1.4) versus (1.8) is highly machine dependent. In many cases (1.8) is more efficient for $N \lesssim 32$, while (1.3)-(1.4) is more efficient for large $N$. However, the form (1.8) is more convenient for the present analysis. Since (1.8) is exact when $u_{N}$ is a trigonometric polynomial of degree less than or equal to $N$, we have

LEMMA 1.1.

$$
\sum_{\substack{k=0 \\ k \neq j}}^{2 N-1} \cos N x_{k} \cos N\left(x_{k}-x_{j}\right) \cot \left(\frac{x_{k}-x_{j}}{2}\right)=0, \quad x_{j}=\frac{j \pi}{N}
$$

Proof. Choosing $u_{N}(x)=\cos N x$, we have by the exactness of (1.8) that

$$
\begin{aligned}
& \sum_{\substack{k=0 \\
k \neq j}}^{2 N-1} \cos N x_{k} \cos N\left(x_{k}-x_{j}\right) \cot \left(\frac{x_{k}-x_{j}}{2}\right) \\
& =-\left.\frac{\partial}{\partial x} \cos N x\right|_{x=x_{j}}=N \sin N x_{j}=0 .
\end{aligned}
$$

This result can also be shown by direct calculation. 
When $c(x)$ is strictly positive (or negative) throughout $[0,2 \pi]$ it is known that the pseudospectral Fourier method gives a stable, consistent, and convergent approximation to (1.1) as $N \rightarrow \infty$ for all $t$ ([2], [3], [11], [13]). On the other hand, when $c(x)$ has a zero within $[0,2 \pi]$ and, in particular, if $c(x)$ changes sign in $[0,2 \pi]$, solutions of (1.5) may grow without bound as $t \rightarrow \infty$. Kreiss and Oliger [8] analyzed the case $c(x)=1-2 \cos x$ and showed that the time derivative of the $L^{2}$-norm of the numerical solution is not bounded by the $L^{2}$-norm of the numerical solution. On the basis of this analysis, Fornberg [2], and Majda, McDonough, and Osher [10] concluded that instabilities as $N \rightarrow \infty$ may occur when $c(x)$ changes sign in the domain. However, as recognized by Kreiss [private communication], the analysis of [8] is not enough to prove instability as $N \rightarrow \infty$.

Here we prove that, for $c(x)=A \sin x+B \cos x+C$ with arbitrary $A, B, C$, (1.5) is stable in the $L^{2}$-norm. Computational evidence is also presented to show that, in general, the source of trouble when $c(x)$ changes sign is not instability as $N \rightarrow \infty$ but rather growth in time. That is, solutions may grow rapidly in $t$ for fixed $N$, but for any fixed $t$ the numerical solution of (1.5) converges as $N \rightarrow \infty$ to the exact solution of (1.1).

Similarly, computational evidence is presented to show that the leapfrog finitedifference scheme provides a stable approximation to (1.1) even though $c(x)$ changes sign.

2. Stability of the Pseudospectral Method. A semidiscrete method is defined to be space-stable (or stable) if for some $T>0$ the solution $u_{N}$ to (1.5) is bounded for $0<t \leqslant T$ for arbitrary initial conditions in some Banach space as the number of mesh points (or modes) $N$ increases. The Lax-Richtmyer equivalence theorem [14] states that if a method is consistent and stable then the numerical solution converges to the solution of the differential equation (1.1).

The method (1.2) is defined as time-stable (or asymptotically stable) if the solution $u_{N}$ to (1.5) is bounded for a fixed number of mesh points (or modes) as $t \rightarrow \infty$. To be precise, attention is restricted to cases where the analytic solution to (1.1) does not grow in time. More generally, time-stability should be defined by the requirement that the solution to the approximate system (1.5) grows no more rapidly than the solution to (1.1) [5].

A necessary and sufficient condition that the approximation

$$
\frac{\partial u_{N}}{\partial t}=Q_{N} u_{N}
$$

be stable is that there exist positive definite matrices $H_{N}$ and finite constants $\alpha_{N}, \beta_{N}$ such that

$$
Q_{H} H_{N}+H_{N} Q_{N}^{*} \leqslant \beta_{N} H_{N}, \quad 0<\alpha_{N} I<H_{N} \leqslant I / \alpha_{N},
$$

where $Q_{N}^{*}$ is the Hermitian adjoint of $Q_{N}$. When $\alpha_{N}$ and $\beta_{N}$ are independent of $N$, the algorithm is space-stable. If $\alpha_{N}=O\left(N^{a}\right)$ [ $a$ finite], $\beta_{N}=O(\log N)$ as $N \rightarrow \infty$, then (2.1) is said to be algebraically space-stable and $u_{N}$ converges to $u$ as $N \rightarrow \infty$ for sufficiently smooth initial data [3]. When $\beta_{N} \leqslant 0$, the scheme is time-stable. 
Consider the equations

$$
\begin{gathered}
u_{t}=[(A \sin x+B \cos x+C) u]_{x}, \quad 0<x \leqslant 2 \pi, t>0, \\
u(0, t)=u(2 \pi, t), \quad u(x, 0)=u_{0}(x),
\end{gathered}
$$

and

$$
u_{t}=(A \sin x+B \cos x+C) u_{x} .
$$

Equations (2.3) are analyzed in this study based on the representation (1.8). (2.3b) contains as a subcase the problem considered by Kreiss and Oliger [8]. An alternative proof of the stability of (2.3b) is presented in [6] based on a representation of the pseudospectral method in Fourier space. There it is shown that one may expect differences in the stability properties of the scheme depending on whether an even number or odd number of collocation points are used.

For the differential equations (2.3) one has an energy estimate

$$
\frac{d}{d t} \int_{0}^{2 \pi} u^{2}(x, t) d x \leqslant(|A|+|B|) \int_{0}^{2 \pi} u^{2}(x, t) d x .
$$

This implies that

$$
\left(\frac{1}{2 \pi} \int_{0}^{2 \pi} u^{2}(x, t) d x\right)^{1 / 2} \leqslant e^{\frac{1}{2}(|A|+|B|) t}\left(\frac{1}{2 \pi} \int_{0}^{2 \pi} u^{2}(x, 0) d x\right)^{1 / 2} .
$$

This energy inequality holds despite the fact that the wave speed $C(x)=A \sin x+$ $B \cos x+C$ may change sign in [0,2 $]$. For the Fourier method (1.2)-(1.5) we shall similarly prove

THEOREM 2.1. Let $u_{N}$ be the pseudospectral Fourier semidiscrete approximation for Eq. (2.3a). Let $2 N \sigma_{N}$ be the last coefficient of the finite Fourier transform of the initial data, i.e.,

$$
\sigma_{N}=\sum_{j=0}^{2 N-1} u_{N}\left(x_{j}, 0\right) \cos N x_{j}, \quad x_{j}=\frac{\pi j}{N}
$$

Then we have the energy inequality

$$
\begin{gathered}
\left(\frac{1}{2 N} \sum_{j=-0}^{N-1} u_{N}^{2}\left(x_{j}, t\right)\right)^{1 / 2} \leqslant \\
\leqslant e^{\frac{1}{2}(|A|+|B|) t}\left(\frac{1}{2 N} \sum_{j=0}^{2 N-1} u_{N}^{2}\left(x_{j}, 0\right)\right)^{1 / 2} \\
+\left|2 \sigma_{N}\right|\left(e^{\frac{1}{2}(|A|+|B|) t}-1\right) .
\end{gathered}
$$

Proof. Using (1.5) and (1.8), we have that

$$
\begin{aligned}
\frac{\partial u_{N}}{\partial t}\left(x_{l}\right)= & \sum_{\substack{j=0 \\
j \neq l}}^{2 N-1}\left(A \sin x_{j}+B \cos x_{j}+C\right) \cos N\left(x_{l}-x_{j}\right) \\
& \times \cot \left(\frac{x_{l}-x_{j}}{2}\right) u_{N}\left(x_{j}\right) .
\end{aligned}
$$


Multiplying (2.7) by $\cos N x_{l}$ and summing over $l$, we have

$$
\begin{aligned}
\sum_{l=0}^{2 N-1} \cos N x_{l} \frac{\partial u_{N}}{\partial t}\left(x_{l}\right)= & \sum_{j=0}^{2 n-1}\left(A \sin x_{j}+B \cos x_{j}+C\right) u_{N}\left(x_{j}\right) \\
& \times \sum_{\substack{l=0 \\
l \neq j}}^{2 N-1} \cos N x_{l} \cos N\left(x_{l}-x_{j}\right) \cot \left(\frac{x_{l}-x_{j}}{2}\right) .
\end{aligned}
$$

Hence, by Lemma 1.1,

$$
\frac{\partial}{\partial t} \sum_{l=0}^{2 N-1} \cos N x_{l} u_{N}\left(x_{l}\right)=0
$$

and so

$$
\sum_{l=0}^{2 N-1} \cos N x_{l} u_{N}\left(x_{l}, t\right)=\sigma_{N}
$$

for all $t$.

We now multiply (2.7) by $u_{N}\left(x_{l}\right)$ and sum over $l$ to get

$$
\begin{aligned}
\frac{1}{2} \frac{d}{d t} \sum_{l=0}^{2 N-1} u_{N}^{2}\left(x_{l}, t\right)= & \frac{1}{2} \sum_{l=0}^{2 N-1} \sum_{\substack{j=0 \\
j \neq l}}^{2 N-1}\left[A \sin x_{j}+B \cos x_{j}+C\right] \\
& \times \cos N\left(x_{l}-x_{j}\right) \cot \left(\frac{x_{l}-x_{j}}{2}\right) u_{N}\left(x_{j}\right) u_{N}\left(x_{l}\right) .
\end{aligned}
$$

We now make use of the identity

$$
\cot \left(\frac{x_{l}-x_{j}}{2}\right)=-\frac{\sin x_{l}+\sin x_{j}}{\cos x_{l}-\cos x_{j}}=\frac{\cos x_{l}+\cos x_{j}}{\sin x_{l}-\sin x_{j}} .
$$

Taking the symmetric part of (2.10), we get

$$
\begin{aligned}
& \frac{1}{2} \frac{d}{d t} \sum_{l=0}^{2 N-1} u_{N}^{2}\left(x_{l}, t\right) \\
&=-\frac{1}{4} \sum_{l=0}^{2 N-1} \sum_{j=0}^{2 N-1}\left[A\left(\cos x_{l}+\cos x_{j}\right) \cos N\left(x_{l}-x_{j}\right)\right. \\
&\left.\quad+B\left(\sin x_{l}+\sin x_{j}\right) \cos N\left(x_{l}-x_{j}\right)\right] u_{N}\left(x_{j}\right) u_{N}\left(x_{l}\right) \\
&-\frac{1}{2} \sum_{l=0}^{2 N-1}\left(A \cos x_{l}-B \sin x_{l}\right) u_{N}^{2}\left(x_{l}\right) .
\end{aligned}
$$

Since $\cos N\left(x_{l}-x_{j}\right)=(-1)^{l+j}$, we also have

$$
\begin{aligned}
\frac{d}{d t} \sum_{l=0}^{2 N-1} u_{N}^{2}\left(x_{l}, t\right)= & -\left(\sum_{l=0}^{2 N-1} u_{N}\left(x_{l}\right) \cos N x_{l}\right) \\
& \times\left(\sum_{j=0}^{2 N-1} u_{N}\left(x_{j}\right) \cos N x_{j}\left(A \cos x_{j}+B \sin x_{j}\right)\right) \\
& -\sum_{l=0}^{2 N-1}\left(A \cos x_{l}-B \sin x_{l}\right) u_{N}^{2}\left(x_{l}\right) .
\end{aligned}
$$


We have, however, the estimate

$$
\begin{aligned}
\sum_{j=0}^{2 N-1} u_{N}\left(x_{j}\right) \cos x_{j} \cos N x_{j} & \leqslant\left(\sum_{j=0}^{2 N-1} u_{N}^{2}\left(x_{j}\right)\right)^{1 / 2}\left(\sum_{j=0}^{2 N-1} \cos ^{2} x_{j}\right)^{1 / 2} \\
& \leqslant N^{1 / 2}\left(\sum_{j=0}^{2 N-1} u_{N}^{2}\left(x_{j}\right)\right)^{1 / 2} .
\end{aligned}
$$

We next define

$$
\left\|u_{N}\right\|^{2}=\frac{1}{2 N} \sum_{j=0}^{2 N-1} u_{N}^{2}\left(x_{j}\right) .
$$

Using (2.8) and (2.11) together with (2.10), we get

$$
\frac{d}{d t}\left\|u_{N}\right\|^{2} \leqslant\left|2 \sigma_{N}\right|(|A|+|B|)\left\|u_{N}\right\|+(|A|+|B|)\left\|u_{N}\right\|^{2}
$$

or

$$
\frac{d}{d t}\left\|u_{N}\right\| \leqslant\left|\sigma_{N}\right|(|A|+|B|)+\frac{1}{2}(|A|+|B|)\left\|u_{N}\right\| .
$$

Using the Gronwall inequality, we conclude that

$$
\left\|u_{N}(t)\right\| \leqslant e^{\frac{1}{2}(|A|+|B|) t}\left\|u_{N}(0)\right\|+2\left|\sigma_{N}\right|\left(e^{\frac{1}{2}(|A|+|B|) t}-1\right) .
$$

COROLlARY 2.1. If the initial data has a continuous first derivative, then the energy inequality (2.6) can be improved to yield

$$
\left\|u_{N}(t)\right\| \leqslant e^{\gamma t}\left\|u_{N}(0)\right\|
$$

where

$$
\gamma \geqslant \frac{1}{2}(|A|+|B|) \frac{2\left|\sigma_{N}\right|}{\left\|u_{N}(0)\right\|}=\gamma_{0} .
$$

Proof. Let $\alpha=\frac{1}{2}(|A|+|B|)$ and $\beta=2\left|\sigma_{N}\right|$. Then (2.7) can be expressed as $\|u(t)\|=e^{\alpha t}\|u(0)\|+\beta\left(e^{\alpha t}-1\right)$. By comparing derivatives, it is easily verified that

$$
e^{\alpha t}\|u(0)\|+\beta\left(e^{\alpha t}-1\right) \leqslant e^{\gamma t}\|u(0)\|,
$$

and (2.15) follows. It only remains to verify that $\gamma_{0}$ is bounded independent of $N$. When $u_{N}(0)$ has a continuous first derivative, then the coefficients of the trigonometric interpolation function $C_{N}$ decay more rapidly than $1 / N$; see, e.g., [15]. Hence, $\beta_{N} /\left\|u_{N}(0)\right\|=2 N C_{N} /\left\|u_{N}(0)\right\| \rightarrow 0$, and, in particular, $\gamma_{0}$ is bounded independent of $N$.

Note 1. If $u_{N}(0)$ is of bounded variation, then $\sigma_{N}$ is bounded as $N \rightarrow \infty$ (see [15, Part II, p. 14]), and therefore convergence still holds. This requirement of bounded variation is reasonable for physically relevant problems.

Note 2. The analysis given above illustrates the idea of low-pass filtering to achieve stability; see [9], [10]. In fact, if $\sigma_{N}=0$ for the initial conditions, then the estimate (2.14) holds. 
COROllary 2.2. For (2.3b), the pseudospectral method (1.5) is stable in the $H^{1}$ norm.

Proof. Let $R$ denote the operation of multiplication by $c(x)=A \sin x+B \cos x$ $+C$ and let $S$ denote differentiation by the pseudospectral algorithm. Then (1.5) becomes

$$
\frac{\partial u_{N}}{\partial t}=R S u_{N}
$$

Defining $v_{N}=S u_{N}$, gives $\partial v_{N} / \partial t=S R v_{N}$, so, noting (2.13),

$$
\frac{d}{d t}\left\|v_{N}\right\| \leqslant(|A|+|B|)\left(\Psi_{N}+\frac{1}{2}\left\|v_{N}\right\|\right)
$$

where

$$
\Psi_{N}=\sum_{j=0}^{2 N-1} v_{N}(x, 0) \cos N x_{j}
$$

Since $v_{N}=S u_{N}$, it is readily verified that $\Psi_{N}=0$, so

$$
\left\|S u_{N}(t)\right\|<e^{\frac{1}{2}(|A|+|B|) t}\left\|S u_{N}(0)\right\| .
$$

From (2.17), it follows that

$$
\frac{1}{2} \frac{\partial}{\partial t}\left\|u_{N}\right\|^{2}=\left(u_{N}, R S u_{N}\right) \leqslant(|A|+|B|)\left\|u_{N}\right\|\left\|S u_{N}\right\|,
$$

so

$$
\frac{\partial}{\partial t}\left\|u_{N}\right\| \leqslant(|A|+|B|)\left\|S u_{N}(0)\right\| e^{\frac{1}{2}(|A|+|B|) t}
$$

Therefore,

$$
\left\|u_{N}(t)\right\| \leqslant\left\|u_{N}(0)\right\|+2\left\|S u_{N}(0)\right\|\left(e^{\frac{1}{2}(|A|+|B|) t}-1\right),
$$

proving $H^{1}$ stability.

3. Results for Spectral Methods. Consider the problem

$$
u_{t}+\sin (\delta x-\gamma) u_{x}=0 \quad(0<x<2 \pi), \quad u(x, 0)=f(x) .
$$

For all the runs $\gamma=\tan (0.9)$ and $\delta=1$, so that $\sin (\delta x-\gamma)$ is not zero at a collcation point. The equation is solved numerically by the Fourier method described in Section 1. The time integration is done with a fourth order RungeKutta method with $\Delta t=1 / 10 N$. Smaller time steps were also tried to determine that errors in the time direction were not contaminating the results. The analytic solution to (3.1) is given by

$$
u(x, t)=f\left(2 \delta^{-1} \tan ^{-1}\left[e^{-\delta t} \tan \left(\frac{\delta x-\gamma}{2}\right)\right]+\gamma \delta^{-1}\right) .
$$

We chose the initial conditions as

$$
f(x)=\frac{\cos x}{1+0.9 \cos \left(2 x+\frac{\pi}{3}\right)},
$$


so that a range of modes are present in the solution. In Table la we present the error, in the standard $L^{2}$-norm, between the exact solution $u(x, t)$, as given by (3.2), and $U$ obtained by the Fourier collocation method. It is evident that the method is converging for sufficiently small $t$. In fact, for any fixed finite $t$ the method converges if sufficiently many modes are used. It is also evident that the error exhibits growth in time. Spectral analysis of the numerical solution shows that only the lowest and highest modes are increasing as shown in [6]. When $\delta=4$ in (3.1), then all the Fourier modes grow in time. In Table $1 \mathrm{~b}$ we present results for the same problem using the formulation suggested in [3]. Thus (3.1) is rewritten as

$$
u_{t}+\frac{1}{2} \sin (\delta x-\gamma) u_{x}+\frac{1}{2}(\sin (\delta x-\gamma) u)_{x}-\frac{\delta}{2} \cos (\delta x-\gamma) u=0
$$

TABLE la

Error for the Fourier collocation method for (3.1) with

$\delta=1, \gamma=\tan ^{-1}(0.9)$ and $f(x)$ given by (3.3).

Here $t$ is measured in units of $2 \pi$

\begin{tabular}{|c|c|c|c|}
\hline$t=N$ & 32 & 64 & 128 \\
\hline .125 & 0.39 & 0.050 & 0.0014 \\
\hline .250 & 0.78 & 0.35 & 0.074 \\
\hline 1.0 & 1.8 & 3.5 & 1.6 \\
\hline 10.0 & 22.8 & 30.2 & 14.4 \\
\hline
\end{tabular}

TABLE $1 \mathrm{~b}$

Same as Table la but for the Fourier collocation method based on (3.4)

\begin{tabular}{|c|c|c|c|}
\hline$t_{t} N$ & 32 & 64 & 128 \\
\hline .125 & 0.23 & 0.035 & 0.00083 \\
\hline .250 & 0.50 & 0.17 & 0.031 \\
\hline 1.0 & 1.9 & 1.4 & 1.0 \\
\hline 10.0 & 25.8 & 9.1 & 11.5 \\
\hline
\end{tabular}

As seen from Table 1b, no substantial improvements over the standard collocation method are observed. There is still space-stability but no time-stability. Other computations were performed with different functions $c(x)$ in (1.1). Even though Theorem 2.1 is no longer applicable the computational conclusion is the same. There is space-stability but generally no time-stability for the Fourier collocation method for equations with variable coefficients. 
TABLE 2

Error for the Fourier collocation method for (3.1) with

$\delta=1, \gamma=\tan ^{-1}(0.9)$, and the discontinuous initial

conditions (3.5)

\begin{tabular}{|c|c|c|c|l|}
\hline \multicolumn{1}{|c|}{$N$} & 32 & 64 & 128 & 256 \\
\hline .125 & 0.63 & 0.65 & 0.61 & 0.63 \\
.250 & 1.0 & 1.1 & 1.0 & 1.1 \\
1.0 & 2.6 & 4.2 & 2.7 & \\
5.0 & 10.0 & 15.6 & & \\
10.0 & 20.0 & 29.4 & & \\
\hline
\end{tabular}

In Table 2, results are presented for $\delta=1$ with the initial conditions

$$
u= \begin{cases}+1, & 0 \leqslant x \leqslant \pi \\ -1, & \pi<x<2 \pi\end{cases}
$$

The results given in Table 2 show that the numerical results are stable but not convergent. In [3] it was shown that this occurs because the Fourier spectral method is not consistent when the solution is not periodic. Analysis of the numerical solution shows that it has many large oscillations.

Additional insight on the nature of the stability of these schemes can be achieved by studying the use of the Chebyshev collocation method [3]. Specifically, consider the problem

$$
\begin{array}{ll}
u_{t}-x u_{x}=0 & (-1 \leqslant x \leqslant 1) \\
u(x, 0)=f(x), & \\
u(-1, t)=f\left(-e^{t}\right), & u(1, t)=f\left(e^{t}\right),
\end{array}
$$

whose analytic solution is

$$
u(x, t)=f\left(x e^{t}\right)
$$

The solutions to be discussed below were computed by the Chebyshev collocation scheme for three sets of initial conditions:

$$
\begin{aligned}
& f(x)=\sin (\pi x), \\
& f(x)=\sin (\pi x / 16), \\
& f(x)=\sin (\pi x / 100) .
\end{aligned}
$$

As shown in [3], good resolution by the Chebyshev spectral expansions requires at least $\pi$ collocation points per wave length of the solution. For (3.7a) there are $e^{t}$ waves within $|x| \leqslant 1$ at time $t$. Hence,

$$
t \simeq \log \frac{N}{\pi}
$$

For collocation using $N$ points, there are no longer enough polynomials to resolve the solution. Corresponding formulas hold for the other initial conditions. For the initial condition (3.7a) with $N=17$, resolution is lost for $t \gtrsim 1.68$, while for 
$N=33$, resolution is lost for $t \gtrsim 2.32$. In Table 3 the computed errors in the Chebyshev solution of (3.5) are listed for the various initial conditions (3.7). The error is measured in the Chebyshev norm

$$
\|U\|^{2}=\sum_{n=0}^{N-1} a_{n}^{2}
$$

where $U(x)=\sum_{n-0}^{N-1} a_{n} T_{n}(x)$, and $T_{n}(x)=\cos \left(n \cos ^{-1} x\right)$ is the Chebyshev polynomial of degree $n$. The results given in Table 3 show that the Chebyshev collocation method is stable for both increasing $N$ and increasing $t$ even though the coefficient of (3.5) changes sign, as proved analytically previously [3]. Any loss of accuracy is attributable to loss of resolution caused by the decrease in the effective wavelength of the solution for large time. Hence, the Chebyshev collocation method has benefits over the Fourier method for problems with variable coefficients.

TABLE 3

Error for the Chebyshev method for (3.6) with smooth

initial data

Initial Condition

$\sin \pi x$

$\sin \pi x / 16$

$\sin \pi x / 100$

\begin{tabular}{|l|c|c|c|c|c|c|}
\hline$t \quad N$ & 17 & 33 & 17 & 33 & 17 & 33 \\
\hline 1 & $2.7 \times 10^{-4}$ & $1.4 \times 10^{-5}$ & $5.8 \times 10^{-7}$ & $3.6 \times 10^{-8}$ & $1.3 \times 10^{-7}$ & $9.0 \times 10^{-9}$ \\
2 & $1.1 \times 10^{-1}$ & $2.6 \times 10^{-4}$ & $4.2 \times 10^{-6}$ & $2.0 \times 10^{-7}$ & $3.8 \times 10^{-7}$ & $2.0 \times 10^{-8}$ \\
3 & $1.4 \times 10^{-1}$ & $8.6 \times 10^{-2}$ & $3.4 \times 10^{-5}$ & $1.6 \times 10^{-6}$ & $7.4 \times 10^{-7}$ & $3.8 \times 10^{-8}$ \\
4 & $1.7 \times 10^{-1}$ & $9.2 \times 10^{-2}$ & $8.7 \times 10^{-4}$ & $2.7 \times 10^{-5}$ & $7.0 \times 10^{-6}$ & $3.4 \times 10^{-7}$ \\
5 & $1.9 \times 10^{-1}$ & $1.2 \times 10^{-1}$ & $1.2 \times 10^{-1}$ & $8.1 \times 10^{-3}$ & $6.4 \times 10^{-5}$ & $3.0 \times 10^{-6}$ \\
6 & $1.5 \times 10^{-1}$ & $8.7 \times 10^{-2}$ & $1.3 \times 10^{-1}$ & $9.7 \times 10^{-2}$ & $9.3 \times 10^{-3}$ & $4.4 \times 10^{-5}$ \\
7 & $1.7 \times 10^{-1}$ & $1.4 \times 10^{-1}$ & $1.3 \times 10^{-1}$ & $1.1 \times 10^{-1}$ & $1.6 \times 10^{-1}$ & $6.0 \times 10^{-2}$ \\
8 & $1.9 \times 10^{-1}$ & $1.1 \times 10^{-1}$ & $2.0 \times 10^{-1}$ & $1.0 \times 10^{-1}$ & $1.2 \times 10^{-1}$ & $1.1 \times 10^{-1}$ \\
\hline
\end{tabular}

Next, (3.5) has been solved by the Chebyshev method with discontinuous initial data

$$
f(x)= \begin{cases}1, & -1<x<0 \\ 0, & 0<x \leqslant 1\end{cases}
$$

If the error is measured in the norm (3.9), the method is both stable and convergent. Plots of the numerical solution display large oscillations that do not decay as $N$ increases. Spectral analysis of the error shows that nearly all the error is concentrated in the highest mode. Hence, although there is no convergence in $L^{2}$, there is convergence in the norm (3.9). It is obvious that weak damping of the high modes in the Chebyshev collocation method will produce good results in $L^{2}$ even for discontinuous data [4]. 
4. Stability of the Leapfrog Finite-Difference Method. A heuristic argument has been given [1] that the leapfrog method is unstable when $c(x)$ oscillates sufficiently rapidly about zero. The example given in [1] applies only to nonlinear instabilities. For linear problems $c(x)$ cannot oscillate with increasing rapidity as the mesh is refined.

Given the differential equation

$$
u_{t}+c(x) u_{x}=0
$$

the leapfrog finite-difference method is given by

$$
U_{j}^{n+1}=U_{j}^{n-1}-\frac{\Delta t}{\Delta x} c\left(x_{j}\right)\left(U_{j+1}^{n}-U_{j-1}^{n}\right) \quad(0<j \leqslant N) .
$$

Equation (4.2) has been solved numerically with time steps chosen so that $(\Delta t / \Delta x) \max |c(x)|=0.1$. In Table 4 the errors are given for the special case (3.1) with $\delta=1$ and the initial conditions (3.3). It is obvious that for $t$ sufficiently small the method is converging quadratically. If $c(x)$ oscillates more rapidly than in this example, then more mesh points will be needed to resolve the solution. However, for sufficiently fine meshes the leapfrog method will still converge. Only when $c(x)$ oscillates with ever decreasing scale as $N \rightarrow \infty$ (which can occur only if $c$ depends on $u$ ) is the convergence doubtful. As was verified by Fornberg [1], the leapfrog method is unstable in time when $c(x)$ changes sign.

TABLE 4

Relative error in the numerical solution of (3.1) obtained by the leapfrog method. Here $\delta=1, \gamma=\tan ^{-1}(.9)$, and

\begin{tabular}{|c|c|c|c|c|}
\hline $\begin{array}{ll}t & N \\
\end{array}$ & 32 & 64 & 128 & 256 \\
\hline .125 & 0.0071 & 0.0018 & 0.00045 & 0.00014 \\
\hline .250 & 0.025 & 0.0067 & 0.0016 & 0.00041 \\
\hline 1.0 & 0.47 & 0.32 & 0.18 & 0.094 \\
\hline 10.0 & 5.3 & 2.7 & 4.0 & 2.8 \\
\hline
\end{tabular}
$f(x)$ is given by (3.3)

TABLE 5

Relative error obtained applying the leapfrog method to

\begin{tabular}{|c|c|c|c|c|}
\hline $\begin{array}{l}t \\
\end{array}$ & 32 & 64 & 128 & 256 \\
\hline .125 & 0.25 & 0.20 & 0.16 & 0.13 \\
\hline .250 & 0.36 & 0.28 & 0.23 & 0.18 \\
\hline 1.0 & 2.2 & 1.9 & 1.4 & 1.2 \\
\hline 10.0 & 11.8 & 17.0 & 6.6 & 12.9 \\
\hline
\end{tabular}

(3.1) with the discontinuous initial condition (3.4) 
In Table 5 the errors are listed when the leapfrog method is used with the discontinuous initial data (3.4). In contrast to the Fourier method, the leapfrog scheme converges in this case also, though the rate of convergence is only $(\Delta x)^{1 / 3}$. As before there is a growth in time.

5. Summary. Both analytic and computational evidence show that Fourier collocation approximation is stable and convergent for the wave equation (1.1) even when the wave speed $c(x)$ changes sign. If the number of modes is fixed and one does long term integrations, then there may be a growth in time. If the initial data is discontinuous then the method is not consistent. Hence, even though the results are spatially stable, there is no convergence.

Similar results hold for the leapfrog method. Even when coefficients change sign the leapfrog method is stable and converges. As before there may be a growth in time. In addition, Kreiss and Oliger [8] have shown that the leapfrog method is susceptible to nonlinear instabilities.

When the wave speed $c(x)$ changes sign there can be a growth in time with the Galerkin-Fourier method. Hence, this is not a disadvantage of the collocation method compared with the Galerkin method.

Department of Mathematics

Tel-Aviv University

Tel-Aviv, Israel

Department of Mathematics

Massachusetts Institute of Technology

Cambridge, Massachusetts 02139

Courant Institute of Mathematical Sciences

New York University

New York, New York 10012

1. B. ForNBERG, "On the instability of Leap-Frog and Crank-Nicolson approximations of a nonlinear partial differential equation,” Math. Comp., v. 27, 1973, pp. 45-57.

2. B. FORNBERG, "On a Fourier method for the integration of hyperbolic equations," SIAM J. Numer. Anal., v. 12, 1975, pp. 509-528.

3. D. Gotrueb \& S. Orszıg, Numerical Analysis of Spectral Methods: Theory and Applications, SIAM Regional Conf. Series in Appl. Math., 1977.

4. D. Gottlieb, L. Lustman \& S. A. Orszag, "Spectral calculations of one dimensional, inviscid compressible flow," SIAM J. Sci. Statist. Comput., Oct. 1981.

5. D. GotTlieb \& E. Turkel, "On time discretizations for spectral methods," Stud. Appl. Math., v. 63, 1980, pp. 67-86.

6. D. Gotrlemb, S. A. Orszag \& E. TURKeL, Stability of Pseudaspectral and Finite Difference Methods for Variable Coefficient Problems, ICASE Report 79-32, 1979.

7. B. GuSTAFSSON, "On difference approximations to hyperbolic differential equations over long time intervals,” SIAM J. Numer. Anal., v. 6, 1969, pp. 508-522.

8. H. O. KREISS \& J. OLIGER, "Comparison of accurate methods for the integration of hyperbolic equations," Tellus, v. 24, 1972, pp. 199-215.

9. H. O. Kreiss \& J. Oliger, "Stability of the Fourier method," SIAM J. Numer. Anal., v. 16, 1979, pp. 421-433. 
10. A. MAdd, J. McDonough \& S. OshrR, "The Fourier method for nonsmooth initial data," Math. Comp., v. 32, 1978, pp. 1041-1081.

11. S. A. OrszaG, "Comparison of pseudospectral and spectral approximations," Stud. Appl. Math., v. 51, 1972, pp. 253-259.

12. S. A. OrszaG, "Spectral methods for problems in complex geometries," J. Comput. Phys., v. 37, 1980, pp. 70-92.

13. J. PASCIAK, "Spectral and pseudo spectral methods for advection equations," Math.Comp., v. 35, 1980, pp. 1081-1092.

14. R. D. Richtmyer \& K. W. Morton, Difference Methods for Initial-Value Problems, 2nd ed., Interscience Tracts in Pure and Appl. Math., No. 4, Interscience, New York, 1967.

15. A. ZYGmund, Trigonometric Series, Cambridge Univ. Press, New York, 1968. 